\title{
REVIEW
}

\section{THE CHALLENGE OF THE PUBLIC COMMUNICATION OF SCIENCE PUBLIC COMMUNICATION OF SCIENCE IN BRAZIL}

\author{
Sophia Karlla Almeida Motta Gallo*
}

Antônio Carlos Teixeira's PhD ${ }^{1}$ thesis sets out concepts and characteristics that can be used to structure graduate programs in public health in Brazil in terms of the public communication of science. The author presents the results of his study within an exploratory, observational and qualitative perspective and contextualizes them within a globalized world where information circulates regardless of frontiers, the speed of their renewal increasing exponentially as a result of the use of new technologies of communication and information.

Paradoxically, strictly scientific information is still circulating. Given this paradox, the coordinators of postgraduate programs are challenged to reflect on the public communication of science even though they may not recognize the need to develop the skills necessary to adapt the language, style, modes of transmission and channels of communication to specific audiences or the need to create processes of communication such as will make systematic input into and feedback from different audiences outside the academic realm possible. It is no coincidence that one of the major difficulties of the coordinators interviewed was to distinguish between scientific Communication, Dissemination and Diffusion of Knowledge.

In relation to the information that comes from the empirical material, the author points out that had no concern in analyzing the meanings and implications. However, highlights that the number of women (18) is higher by $16 \%$ that the number of men (13); and between men and women varies from 43 to 69 years.

According to the survey data, most coordinators (52\%) are graduated in medicine, and only two coordinators, who have graduation one in Social Sciences and the others in Mathematics and Statics were not more directly related to the health field at graduation studies.

In his study, the author did not have as its object of analysis the time period which the coordinators of postgraduate programs are in the position of administrative leadership, either the time in number of years of existence/recognition programs. While highlighting the fact that three programs were recognized for having more than 30 years, seven programs were recognized for having less than 20 years.

Based on content analysis, the author sought to highlight the concepts that the coordinators of postgraduate programs have public communication of science. And get the following results:

The public communication of science understood as a communication should be extended to the whole of society is the predominant (15 responses). However, restricting the public communication of science is communication among peers (10 responses). According to the author, the narrative of the interviewees does not show the significant fact that the extensive communication to society should be expresses in a language that allows the understanding of the lay public. In the words of him, "that is when the laity is able to decode scientific information that can begin to gain importance".

Although the response of just one of the deponents could not be included in any of the three conceptions of public communication of science, it was considered significant because it reinforced the result of the analysis of the responses previously presented in categories one and two, emphasizing the idea that apparently PHGP coordinators do not have a conception of public communication of science more concatenated to the concept of knowledge society and scientific culture.

For him, the analysis of responses allowed to understand this type of communication as a code translation of scientific language into a language accessible directed to the whole society.

From the results of his doctoral research that discusses the public communication of science, the author hopes that the results may contribute to the discussion of this peculiar form of science

\footnotetext{
1 Post-Doutoral student in Health Sciences (Laboratório de Delineamento de Estudos e Escrita Científica/FMABC) and Researcher of Centro de Estudos do Crescimento e do Desenvolvimento Humano (CDH).
} 
communication in PHGP: "In Brazil there are 54 graduate programs public Health recognized by CAPES that are offered in 35 higher education institutions, 29 public universities and six private institutions. These programs are offered 36 courses MSc, 21 PHD and 15 MSc professional. Such is the magnitude of the problem."

This study reveals that the urgent mission of the university should be understood as a dialogue with society, although, as the author says, there is no consensus among the Coordinators of the PPGSC studied as to what scientific communication is. Some see it as a diffusion of knowledge, without distinguishing between the scientific community and society in general. Others conceive it as the dissemination of knowledge addressed to the

\section{REFERENCE}

1. Teixeira CA (2013). The public communication of Science in graduate programs in public health scientific community while still others understand it in its broader sense, as communication addressed to society as a whole.

What has come to light as a result of this research is that the actions related to scientific communication and the transmission of scientific knowledge are in fact mainly directed to the scientific community itself and are the activities to which the post-graduate program coordinators dedicate most of their time and resources. This study permits one to see that despite the conception of scientific communication targeted to society and despite some of the rhetoric of some coordinators, not everyone understands that this really represents the social role of the university.

in Brazil: a perspective of coordinators. [PhD thesis]. São Paulo: Faculdade de Saúde Pública da USP. 220p. 